\title{
Novel Type of Dislocation in an Al-Pd-Mn Quasicrystal Approximant
}

\author{
H. Klein,* M. Feuerbacher, P. Schall, and K. Urban \\ Institut für Festkörperforschung, Forschungszentrum Jülich GmbH, D-52425 Jülich, Germany
}

(Received 1 December 1998)

\begin{abstract}
A single crystal of the $\xi^{\prime}$-AlPdMn phase was plastically deformed in a three-point bending geometry. The deformed crystal shows a periodic lattice of phason lines. In a high-resolution electron microscopy (HREM) study a novel dislocation type, termed metadislocation, was observed for the first time. The magnitude of its Burgers vector is $171 \AA$. At the core of the metadislocation a dislocation of the $\xi^{\prime}$ structure is found. For the first time its structure and Burgers vector were determined by HREM. In magnitude and direction it is equivalent to the most common Burgers vector in the icosahedral quasicrystal. The structural relationship between the dislocation and the metadislocation is discussed. [S0031-9007(99)08984-X]

PACS numbers: 61.72.Ff, 61.16.Bg, 61.44.Br, 62.20.Fe
\end{abstract}

The predominant plastic deformation mechanism in crystalline solids is governed by the motion of dislocations. Despite the lack of translational symmetry in quasicrystals dislocations exist in them [1], and as in crystals moving dislocations carry plastic flow. This has been shown in high temperature deformation experiments for quasicrystals of icosahedral [2] and decagonal [3] structure. The structure of a quasicrystal, quasiperiodic in the physical three-dimensional space, can be described as a periodic structure in a higher dimensional hyperspace, which is a six-dimensional (6D) space in the case of an icosahedral quasicrystal. The Burgers vectors of dislocations in such a phase can then be described as $6 \mathrm{D}$ vectors. In contrast to periodic crystals, however, icosahedral quasicrystals show no work hardening. They even become softer the more they are plastically deformed [4]; i.e., the flow stress decreases with increasing strain.

In the compositional vicinity of quasicrystalline phases one often finds periodic phases with large unit cells, having cell parameters that can be expressed as a function of the hyperspace cell parameter associated with the corresponding quasicrystal and the golden mean $\tau=(1+\sqrt{5}) / 2$. These phases, termed approximants, are periodic in structure and therefore crystals, and they are close in chemical composition, local order, and physical properties (electrical and thermal conductivity, magnetic behavior, etc.) to quasicrystals [5]. One should therefore also expect to find a similar behavior of approximants regarding the plastic deformation mechanism. On the other hand, due to the large cell parameters in approximants, a complete dislocation in such a phase would necessitate a Burgers vector of more than $10 \AA$ in magnitude. Such a large Burgers vector would involve very high defect energies compared to what is observed in simple crystals, making its existence improbable.

As yet, no experimental investigations of the plastic deformation behavior of approximants have been published. Moreover, no observations of dislocations in approximants have been reported. Klein et al. [6] proposed a geometrical model for the plastic deformation of an approximant $\left(\xi^{\prime}\right.$ phase [7]) of the icosahedral Al-Pd-Mn phase which does not involve dislocations. The model employs a new kind of linear defect which can move under an applied stress and carry plastic flow. The response of this complex intermetallic phase to an external stress is a change of shape as well as a structural change.

In this paper we present the results of a high-resolution electron microscopy (HREM) study of a dislocation observed in a plastically deformed sample of the $\xi^{\prime}$-AlPdMn approximant. Its Burgers vector is shown to be equivalent in magnitude and direction to the most commonly observed Burgers vector in the icosahedral phase. A second type of line defects, so-called phason lines, can arrange periodically. The interaction of a dislocation with the periodic lattice of phason lines leads to a dislocation on another length scale, which is presented here for the first time.

The $\xi^{\prime}$ phase, an approximant of the icosahedral AlPdMn phase, has an orthorhombic unit cell of space group Pnma with cell parameters $a=23.54 \AA$, $b=$ $16.57 \AA$, and $c=12.34 \AA$ [8]. Orientation relationships with the quasicrystal are such that the $\mathbf{b}$ axis is parallel to a fivefold axis, the $\mathbf{c}$ axis is parallel to a twofold axis, and the $\mathbf{a}$ axis is perpendicular to a mirror plane of the icosahedral phase. HREM at an incident electron beam parallel to the b axis of this structure shows flattened hexagons as the salient structural feature. Figure 1(a) shows the arrangement of these hexagons in rows of two different orientations as observed in the undeformed reference sample. The contrasts on the hexagon vertices have been shown to correspond to columns of clusters of icosahedral symmetry containing 51 atoms - the so-called pseudo-Mackay icosahedra [9].

In the geometrical model of plastic deformation of the $\xi^{\prime}$ phase proposed by Klein et al. [6], defects can be introduced into the perfect lattice of flattened hexagons. In this lattice the defects can be characterized by a bananashaped polygon attached to a regular pentagon [labeled " $A$ " in Fig. 1(b)]. They can be introduced at the crystal surface, and they can move parallel to the c direction through successive jumps of hexagon vertices. Note that 


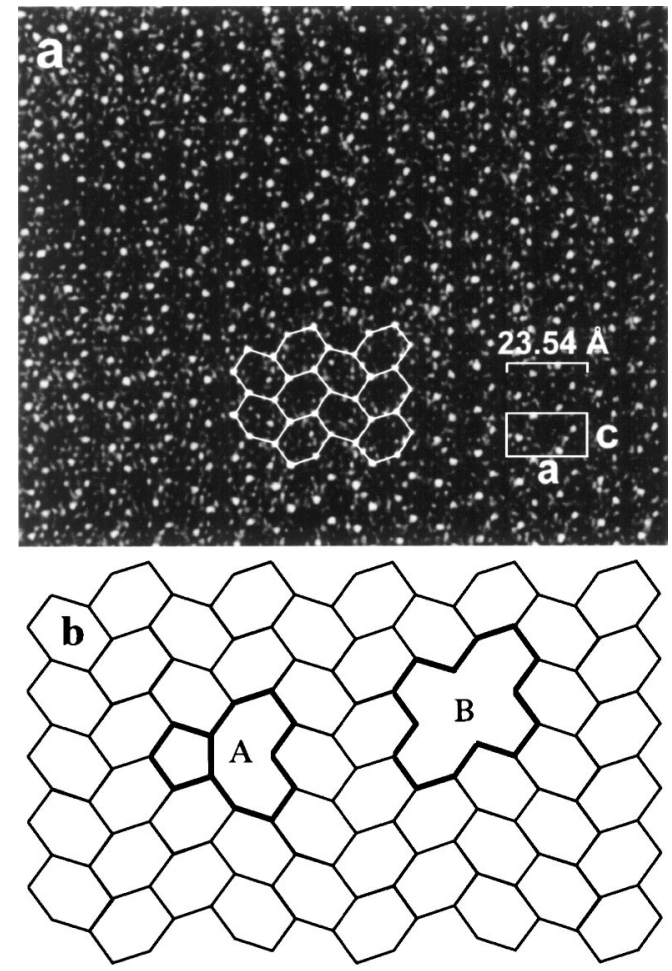

FIG. 1. (a) HREM image of the $\xi^{\prime}$-AlPdMn phase seen along its b axis. Bright contrasts are observed on the vertices of flattened hexagons arranged in rows at two different orientations. (b) Tiling of flattened hexagons with structural defects. A banana-shaped polygon and an attached pentagon (labeled $A$ ) correspond to a phason line with the line direction parallel to $\mathbf{b}$. A phason line including a kink (labeled $B$ ) in the defect line results in an elongation of the defect in the tiling.

these defects are defect lines; i.e., they are extended parallel to the $\mathbf{b}$ axis that is perpendicular to the image plane. The defect lines are not always strictly parallel to $\mathbf{b}$ but can show kinks along the defect line leading to elongated defect contrasts in HREM images [labeled " $B$ " in Fig. 1(b)]. They have been termed phason defects in reference to phason defects in quasicrystals, because the atomic jumps involved in the motion of a defect result in a local rearrangement of the structural building blocks while leaving the structure unchanged at large distances [10]. In the following we will refer to the defects as phason lines in order to emphasize their linear character. Note that the orientation of the flattened hexagons is changed in two rows below the phason line in Fig. 1(b) which corresponds to the trace of the moving phason line. This leads to a local structure change resulting in two neighboring rows of parallel hexagons, which is the characteristic of the $\xi$ phase, another approximant of the icosahedral Al-Pd-Mn phase [7]. In the geometrical model proposed by Klein et al. [6] it has been shown that the successive motion of several phason lines leads to plastic deformation as well as a structural transition from the $\xi^{\prime}$ to the $\xi$ phase.

The sample investigated in the present study is a single crystal of the $\xi^{\prime}$ phase obtained by Bridgman growth. The sample was plastically deformed at $750{ }^{\circ} \mathrm{C}$ under creep conditions, i.e., at constant load, in three-point bending geometry. The orientation was chosen with the $\mathbf{b}$ axis parallel to the bending axis and the c axis parallel to the applied force. The sample size was approximately $4.5 \mathrm{~mm} \times 1.8 \mathrm{~mm} \times 1.2 \mathrm{~mm}$ parallel to $\mathbf{a}, \mathbf{b}$, and $\mathbf{c}$, respectively. Slices perpendicular to the $\mathbf{b}$ axis were cut by a high-precision wire saw and thinned for transmission electron microscopy by mechanical grinding, polishing, and subsequent ion milling on a liquid-nitrogen-cooled stage.

HREM observations on the deformed sample revealed the presence of a periodic arrangement of phason lines, changing the periodicity in the $\mathbf{c}$ direction from $12.34 \AA$ in the original structure to $(\tau+3) \times 12.34 \AA=57 \AA$ in the deformed crystal [Fig. 2(a)]. Thus, a structural transition has taken place during plastic deformation by the creation and motion of phason lines. Electron diffraction patterns obtained with the incident beam parallel to the $\mathbf{b}$ axis confirm the long-range periodic order of the phason lines. The periodicities of the $\mathbf{a}$ and $\mathbf{b}$ directions remain unchanged. Dislocations were also observed in the deformed sample. The line directions of all dislocations are parallel to the b axis yielding endon contrasts when the electron beam is parallel to the $\mathbf{b}$ axis. From Bragg contrast extinction conditions in different two-beam cases the Burgers vectors were determined to be parallel to the $\mathbf{c}$ axis. All dislocations are therefore pure edge dislocations, a circumstance which allows one to determine the magnitude of the Burgers vectors by drawing a Burgers circuit on HREM images taken along the direction of the dislocation line.

In the periodic lattice the phason lines are arranged in horizontal planes perpendicular to the $\mathbf{c}$ axis of the $\xi^{\prime}$ structure, i.e., the only possible direction of motion for the phason lines. In two neighboring planes the phason lines occupy alternating positions, which results in a centered rectangular lattice. The periodic lattice of phason lines itself contains structural defects. Figure 2(b) shows a dislocation in the lattice of phason lines where six halfplanes of phason lines are inserted on the left-hand side. In the following we will term this defect a metadislocation. In the metadislocation core the perfect tiling of flattened hexagons corresponding to the undeformed $\xi^{\prime}$ phase is observed. Drawing a Burgers circuit around the core of the metadislocation a Burgers vector can be defined that is parallel to the $\mathbf{c}$ axis of the $\xi^{\prime}$ structure and whose magnitude is equal to $171 \AA$ corresponding to 3 times the lattice parameter of the phason line lattice.

The observation of a periodic lattice of phason lines provides strong evidence for the existence of interaction forces between the phason lines. The existence of a metadislocation with inserted horizontal half-planes premises an attractive interaction between the phason lines in the a direction of the $\xi^{\prime}$ structure. The interaction between phason lines in neighboring planes must be less attractive or could even be repulsive. In the latter case the lattice would be formed by an external pressure. These types of interactions could 


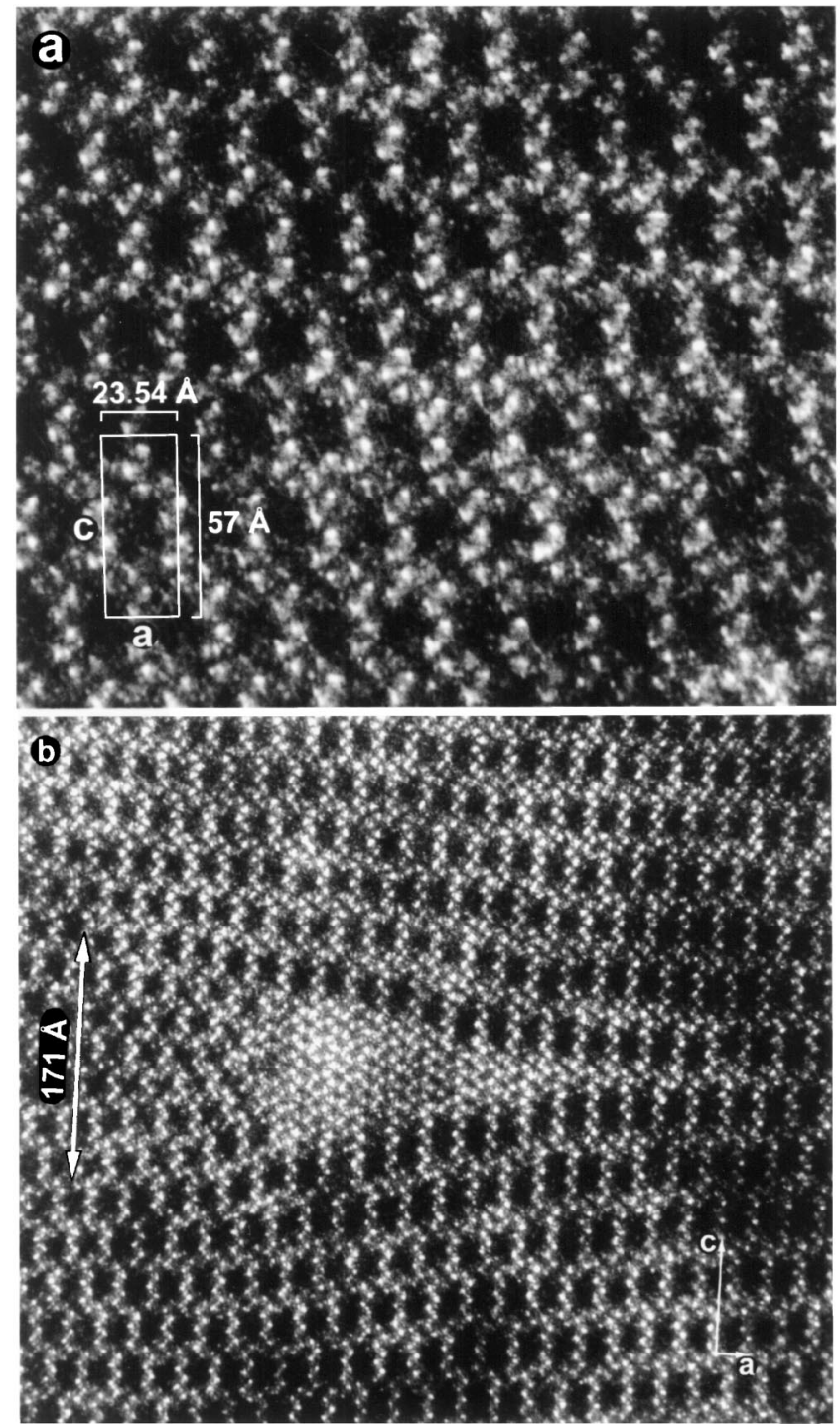

FIG. 2. (a) HREM image of the plastically deformed $\xi^{\prime}$ phase seen along $\mathbf{b}$, where bright contrasts are identical to the contrasts at the hexagon vertices in Fig. 1(a) and dark areas represent phason lines. The phason lines form a centered rectangular lattice. (b) A metadislocation in the lattice of phason lines. The Burgers vector is parallel to $\mathbf{c}$ and its magnitude is $171 \AA$.

be indicated by the shape of the phason lines around the metadislocation. The phason lines observed are not strictly parallel to the $\mathbf{b}$ axis but are elongated in the $\mathbf{c}$ direction. This is due to kinks in the phason line, the number of kinks determining the extension along $\mathbf{c}$. On the side of the inserted half-planes, where due to the stress field around the metadislocation the compression in the phason line lattice is larger, the phason lines are denser and the number of kinks per phason line is smaller than in the undisturbed lattice. On the opposite side of the metadislocation, where its stress field results in tension, the spacings between planes of phason lines and the number of kinks in each phason line is larger.
By drawing the tiling of hexagons, banana-shaped polygons and pentagons in the metadislocation core one observes that these three tiles are not sufficient to cover all the plane near the six inserted half-planes [Fig. 3(a)]. A polygon that cannot be covered by the three tiles remains. A Burgers circuit drawn around this remaining polygon does not close, it reveals a Burgers vector parallel to the $\mathbf{c}$ direction with a magnitude equal to $(2 \tau-3) d=1.83 \AA$, where $d=7.76 \AA$ is the edge length of the tiles. This is precisely the length of the physical space component of the most frequently observed Burgers vector in the plastically deformed icosahedral Al-Pd-Mn phase [11,12]. In the icosahedral phase this Burgers vector is observed to be parallel to a twofold axis which is parallel to the $\mathbf{c}$ axis of the $\xi^{\prime}$ phase according to the orientation relationships.

The polygon characterizing the dislocation core can be embedded in a tiling of flattened hexagons if one allows rows of parallel hexagons [Fig. 3(b)]. On one side of the dislocation three rows of hexagons are parallel; on the other side there are five parallel rows. Using the bananashaped and pentagon tiles corresponding to a phason line one can modify the tiling around the dislocation core in such a way as to regain a perfect tiling of rows of hexagons in alternate orientations [Fig. 3(c)]. For this, six half-planes of phason lines are necessary, just like those observed in HREM. Outside the six half-planes the original structure is restored. Entire planes of phason lines can therefore be built again.

The dislocation can move in the $\mathbf{c}$ direction of the $\xi^{\prime}$ structure according to the shear stress component exerted in the three-point bending geometry. Note, however, that the motion of the dislocation resembles the motion of a phason line. As a phason line moves through the crystal parallel to the $\mathbf{c}$ axis it changes the orientation of two rows of flattened hexagons thus creating two rows of parallel hexagons. The dislocation core moving along the c direction changes the orientation of three rows of hexagons. Starting from the three parallel rows in front of the dislocation core, five parallel rows are generated and plastic flow is transported. The motion of the dislocation therefore has a phasonic character and is closely related to the approximant relationship of the $\xi^{\prime}$ phase to the quasicrystal.

The observed dislocation is a partial dislocation with a Burgers vector whose magnitude is an irrational fraction of the cell parameter $c=12.34 \AA$. This is achieved in the material by a rearrangement of the basic structural units, i.e., pseudo-Mackay icosahedra. In this way a dislocation with a Burgers vector much smaller than the cell parameter can be incorporated in the crystal without changing the local order. In contrast, partial dislocations in simpler structures, e.g., simple ordered intermetallics, massively change the local order, which results in the formation of stacking faults.

In conclusion, we identified a dislocation in the $\xi^{\prime}$ approximant of the Al-Pd-Mn icosahedral phase. Burgers 

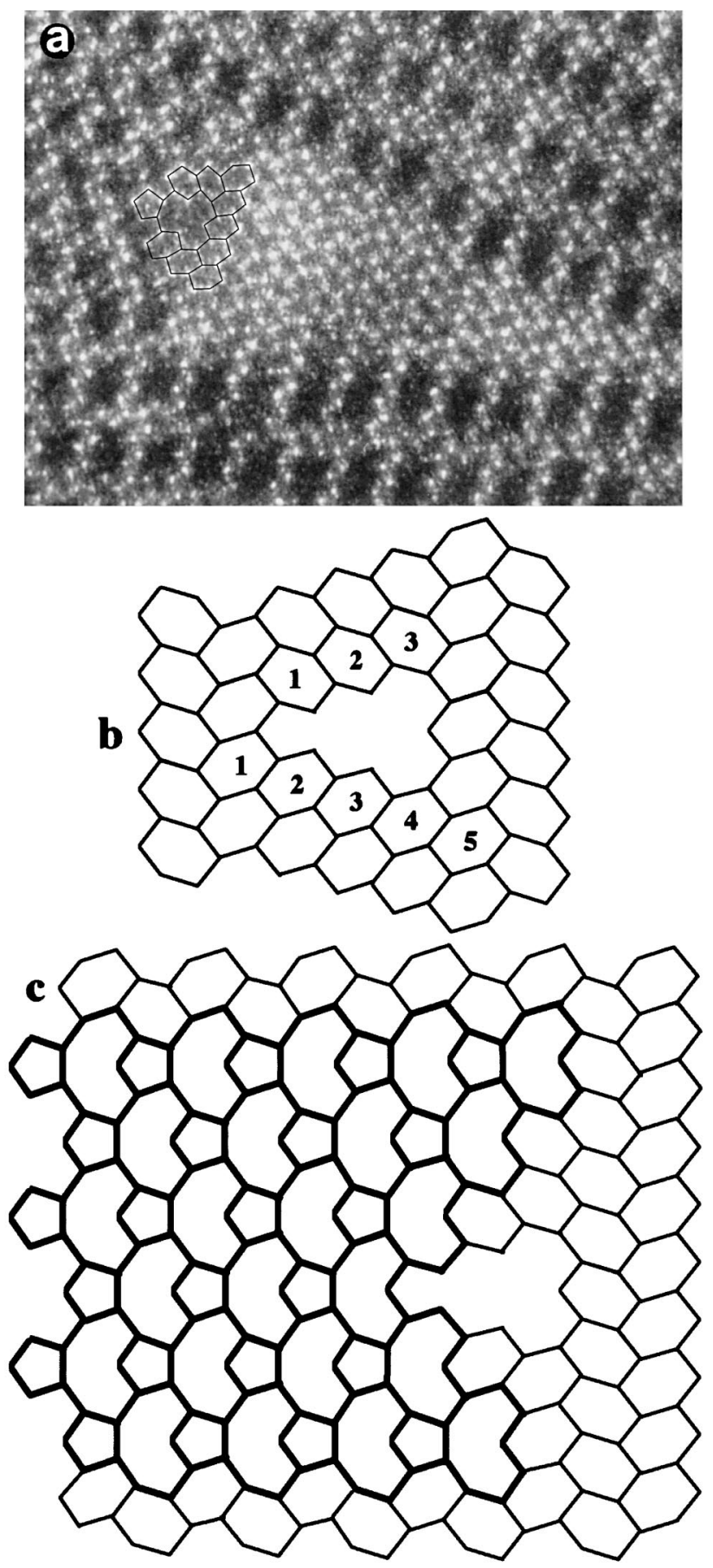

FIG. 3. (a) HREM image taken along b of the core region of the metadislocation. In the center the polygon outlined in black lines cannot be tiled using flattened hexagons, bananashaped polygons, and pentagons. (b) The polygon observed in the core of the metadislocation can be embedded in a tiling of flattened hexagons if one allows for rows of parallel hexagons. On one side three parallel rows are needed, on the other side five. (c) The alternate orientations of flattened hexagons can be recovered above and below the defect by introducing six halfplanes of phason lines, which is consistent with the observation in Fig. 2(b). vector direction and magnitude were determined by HREM of the dislocation viewed endon. The Burgers vector of the dislocation in the approximant is identical in magnitude and, via the orientation relationships between the approximant and the icosahedral phase, in direction to the most frequently found Burgers vector in the icosahedral phase. The dislocation motion in the approximant is driven by the shear stress component it is subjected to in the threepoint bending. Nevertheless, its motion has a phasonic character.

In the periodic lattice of phason lines observed in the plastically deformed $\xi^{\prime}$ phase this dislocation is a nucleation site of a structural defect in the phason line lattice. This metadislocation was observed to have six inserted half-planes of phason lines leading to a Burgers vector of $171 \AA$. Related metadislocations with different numbers of inserted half-planes corresponding to different dislocations of the $\xi^{\prime}$ structure were also observed. These and the structural relationships among this new class of defects will be described elsewhere.

* Present address: European Synchrotron Radiation Facility, Pluo E 202, B.P. 220, 38043 Grenoble Cedex France.

[1] Z. Zhang and K. Urban, Philos. Mag. Lett. 60, 97 (1989); J. Devaud Rzepski, M. Cornier-Quiquandon, and D. Gratias, in Proceedings of the IIIrd International Conference on Quasicrystals (World Scientific, Singapore, 1989), p. 498; S. Ebalard and F. Spaepen, J. Mater. Res. 5, 62 (1990).

[2] M. Wollgarten, M. Beyss, K. Urban, H. Liebertz, and U. Köster, Phys. Rev. Lett. 71, 549 (1993).

[3] M. Feuerbacher, M. Bartsch, B. Grushko, U. Messerschmidt, and K. Urban, Philos. Mag. Lett. 76, 369 (1997).

[4] S.S. Kang and J.M. Dubois, Philos. Mag. A 66, 151 (1997).

[5] C. Janot, Quasicrystals, A Primer (Clarendon Press, Oxford, 1992).

[6] H. Klein, M. Audier, M. Boudard, M. de Boissieu, L. Beraha, and M. Duneau, Philos. Mag. A 73, 309 (1996).

[7] M. Audier, M. Durand-Charre, and M. de Boissieu, Philos. Mag. B 68, 607 (1993).

[8] M. Boudard, H. Klein, M. de Boissieu, M. Audier, and H. Vincent, Philos. Mag. A 74, 939 (1996).

[9] M. Boudard, M. de Boissieu, C. Janot, G. Heger, C. Beeli, H. Nissen, H. Vincent, R. Ibberson, M. Audier, and J. M. Dubois, J. Phys. Condens. Matter 4, 10149 (1992); C. Janot, Phys. Rev. B 53, 181 (1996).

[10] L. Beraha, M. Duneau, H. Klein, and M. Audier, Philos. Mag. A 76, 587 (1997).

[11] M. Feuerbacher, C. Metzmacher, M. Wollgarten, B. Baufeld, M. Bartsch, U. Messerschmidt, and K. Urban, Mater. Sci. Eng. A 233, 103 (1997).

[12] R. Rosenfeld, M. Feuerbacher, B. Baufeld, M. Bartsch, U. Messerschmidt, M. Wollgarten, G. Hanke, M. Beyss, and K. Urban, Philos. Mag. Lett. 72, 375 (1995). 